\title{
A Requirements Evaluation Method for Ships to Maximize Operational Value under Uncertainty
}

\author{
Kazuo Hiekata ${ }^{1}$ and Bryan Moser $^{2}$ \\ ${ }^{1}$ Graduate School of Frontier Sciences, The University of Tokyo, Japan \\ ${ }^{2}$ Engineering Systems Division, M.I.T. and Global Project Design, USA \\ hiekata@k.u-tokyo.ac.jp, bry@mit.edu
}

\begin{abstract}
Requirements defined by shipping firms play a role as the communication interface of product information between these firms and shipbuilders, and the product design is optimized based on the requirements. Ship service life is about 30 years, during which original requirements may lose some relevance. In this paper we explore the introduction of uncertainty and flexibility into requirements so that ships are designed optimally not only for a single given technical condition but for a range of possible operational scenarios, including shifts in the economic environment for shipping firms.
\end{abstract}

\section{Introduction}

\subsection{Background}

Shipping firms design their business plans and define the requirements for vessels to be newly acquired. The requirements include essential performance and constraints depending on planned routes and their forecasted demand. Therefore the characteristics of the vessels will be optimized for the specific requirements based on the shipping firm's strategy. Shipbuilders then proceed with basic design work to satisfy these requirements and constraints such as cost, delivery, performance and other dependencies.

From a shipping firm's point of view, the basis to measure performance of vessels will be different from the shipbuilders'. At the delivery of vessels, shipbuilders and shipping firms must find some common criteria for evaluation. Today, key performance characteristics are evaluated under standard protocols. Classification societies check the structural performance and the propulsive performance as evaluated in sea trials. During these propulsive performance evaluations, the protocol for sea trial considers only calm sea conditions, even though actual operation might occur across a large variation of sea conditions. Nominally, a shipbuilder can optimize the design of a vessel for calm seas at a given service speed, and the ship might demonstrate good performance in severe weather.

Recently shipping operations are in need of better fuel consumption rate and reduction of greenhouse gas (GHG) emissions. For example, the "10 Mode Performance Index for Ships [1]" was developed to evaluate the decreases in service speed at a 
given power rating for the predefined sea conditions. The development and deployment of this kind of index may expose the performance of vessels in various conditions which were not explored in the trials held at calm seas.

As mentioned above, requirements defined by shipping firms play a role as the communication interface of product information between these firms and shipbuilders, and the product design is optimized based on the requirements. Ship service life is about 30 years, during which original requirements may lose some relevance. In this paper we explore the introduction of uncertainty and flexibility into requirements so that ships are designed optimally not only for a single given technical condition but for a range of possible operational scenarios, including shifts in the economic environment for shipping firms.

\subsection{Objective}

This research proposes the requirements evaluation method to maximize the benefit of ships in the operation. The key idea is to consider the changes in economic conditions in addition to the optimization for a given design requirement.

\section{Proposed Method}

\subsection{Overview}

The performance in fuel consumption rate is governed by the resistance of the ship hull in operation. Generally the resistance by the sea water increases for larger service speed as shown in Figure 1. Froude Number $\left(F_{n}\right)$ is defined as the ratio of a characteristic velocity to a gravitational wave velocity and Reynolds number $\left(R_{n}\right)$ is defined as the ratio of inertial forces to viscous forces. The design service speed is defined by ship owners to fit the planned route for the vessel and the design of the vessels is optimized for the service condition, therefore the main engine and propeller demonstrate optimal performance at the condition.

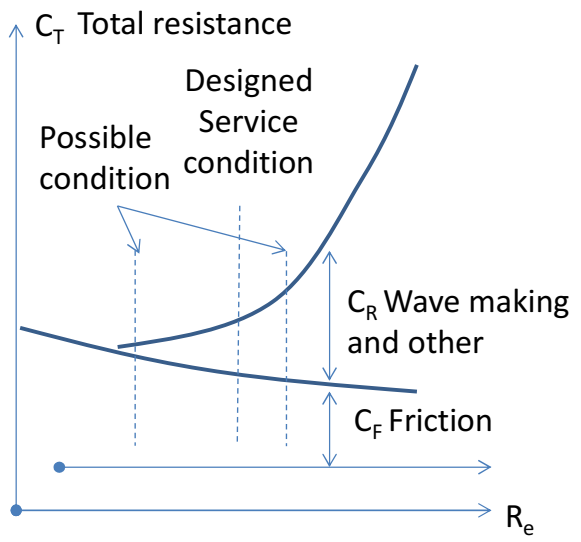

Fig. 1. Resistance of ship at various conditions 
Though the fuel consumption rate is optimized at the given speed, the shipping firms will operate their ships at faster or slower speed to maximize the profit or minimize the loss in uncertain future economic situations during the service life of the vessels.

The proposed method calculates the performance at designed service conditions and possible various conditions to support the evaluation of requirements definition.

\subsection{Fuel Consumption Model}

The fuel consumption is the outcome of the interactions of the main engine, the propeller and the hull. The characteristics of these factors are nonlinear and the design of the plant is usually optimized for reducing the fuel consumption rate at the service speed which is assumed to be most likely at the acquisition. The fuel consumption model is based on the systematized ship design theory such as [2].

The ship hull is the major source of the resistance. Wave, viscous and viscous pressure resistances gives large effects on the performance. The total resistance of the ship hull will be calculated by the results of towing tank tests. The total resistance will be separated to friction and residual resistances. The friction has significant impact on slow vessels and the residual on the faster. Each type of resistance will be calculated as non-dimensional coefficients depending on Froude number and Reynolds number. The Froude number are about 0.15 for slow vessels such as tankers and about 0.27 for the faster such as container ships. The resistance is usually described as EHP (Effective Horse Power) at any ship speeds.

The output of the main engine is given by the rotation speed and the torque delivered from the shaft. BHP(Brake Horse Power) indicates the power generated by the main engine. Engines generally have their optimal load in the specific fuel consumption per output. A typical example is shown in Figure 2. Detailed behavior of the engine may be found in [3]. The power generated by the main engine will be transferred to the propeller with a certain amount of losses.

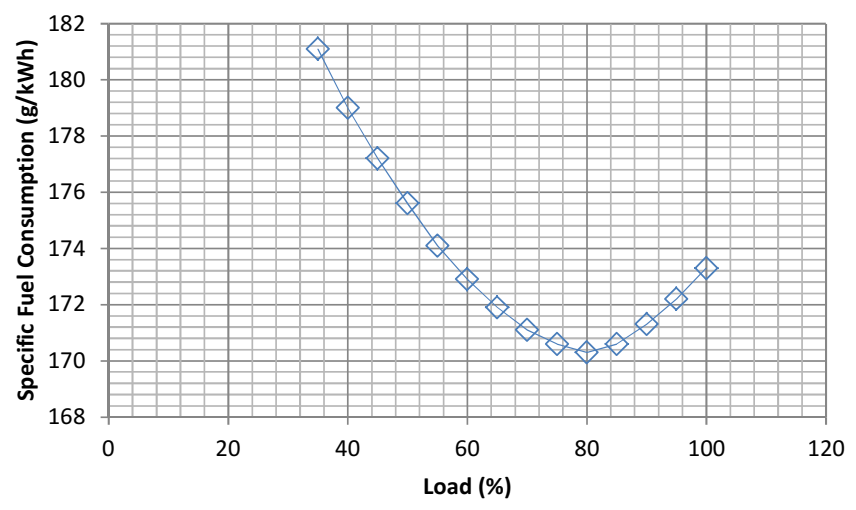

Fig. 2. Load and efficiency of a main engine 
Finally, propellers will give the thrust to the ship hull through the use of the torque delivered from the shaft. The condition of the propeller in operation must be defined to calculate the performance of propellers. The performance of propellers is normally described like a graph shown in Figure 3. $\mathrm{J}$ is advanced coefficient and given by the following equation. $\mathrm{V}_{A}$ is advanced velocity into the propeller, $\mathrm{K}_{T}$ is thrust coefficient given by open water test and $K_{Q}$ is propeller torque coefficient. Ship designers usually try to maximize the propeller efficiency $\eta_{0}$.

$$
\mathrm{J}=\frac{V_{A}}{n D}
$$

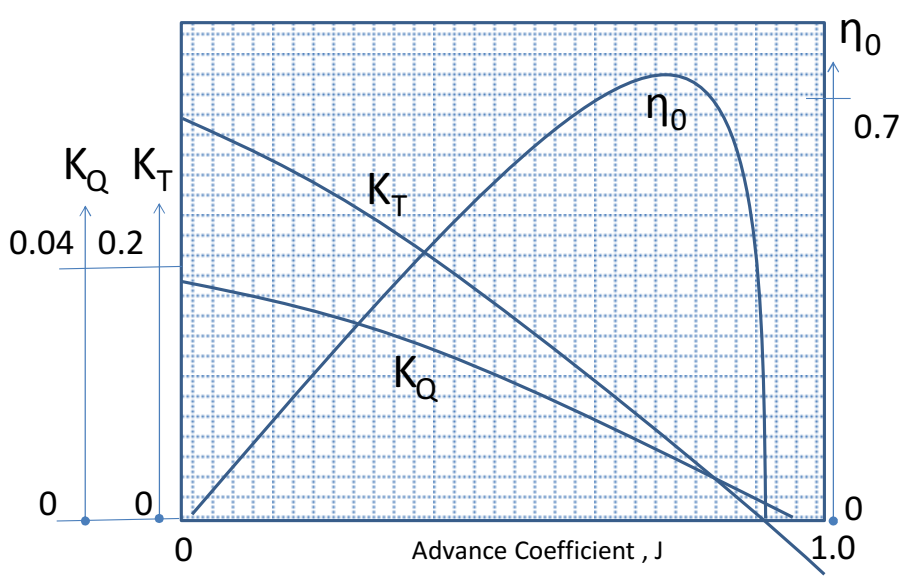

Fig. 3. Characteristics of a propeller

The service speed is defined in the requirement for a specific vessel, and then the propeller and the main engine will be configured. The design process will be iterated to improve the design of the vessel. The efficiency of ships is optimized at the specified service speed. The fuel consumption rate for other service speed can be calculated based on the characteristics of main engine, propeller and hull.

\subsection{Marine Transportation Cost and Revenue Model}

The forecast of the oil price is required to predict the ship service speed for maximizing the profit rate per oil consumption or the amount of the profit. The historical data of the oil price is shown in Figure 4. According to the fluctuation of the oil price, the vessels should be designed for varieties of the market situation.

The shipping market for oil tankers are described by using Worldscale flat rate issued by Worldscale association (LONDON) limited and Worldscale association (NYC) inc. The flat rate is defined as a tariff based on standard tankers in USD/metric ton for each shipping route. For example, the route from Ras Tannurah, Saudi Arabia to Chiba, Japan has the flat rate. The negotiations in shipping market are based on this Worldscale flat rate, and the rate for a specific route will be defined as the rate in 
Worldscale. If the rate for the route from Ras Tannurah, Saudi Arabia to Chiba, Japan is 50 percent (WS50) and the flat rate (WS100) is $20 \mathrm{USD} / m e t r i c$ ton, the freight will be $10 \mathrm{USD} /$ metric ton, 50 percent of flat rate. The flat rates for all the routes in the world are published on January 1 and July 1 every year. As Worldscale flat rate is calculated based on the oil price, it has strong correlation with the oil price, so the flat rate can be calculated by given oil price. The historical data is shown in Figure 5.

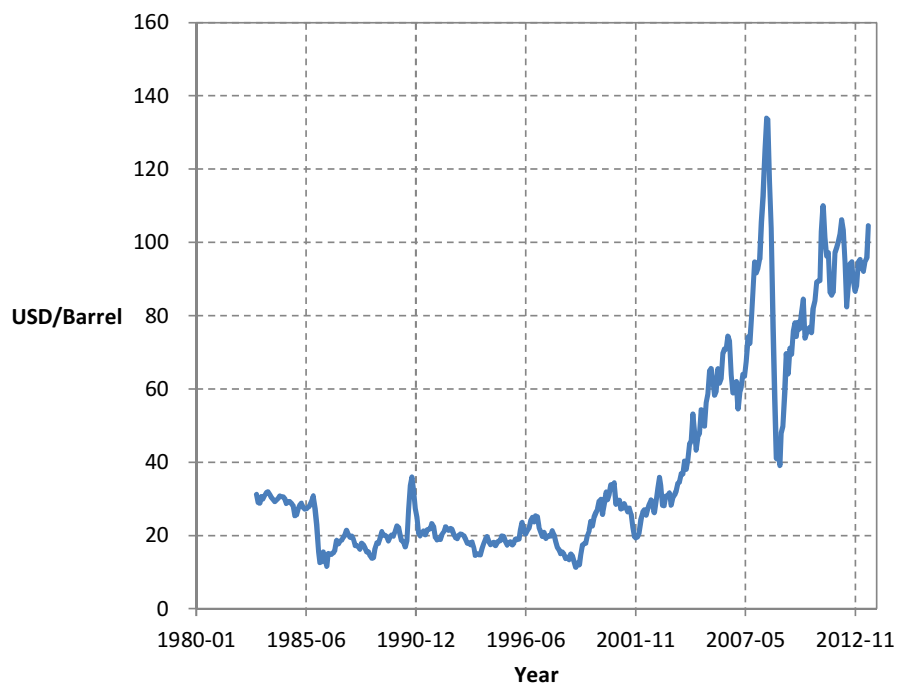

Fig. 4. Spot Oil Price West Texas Intermediate [4]
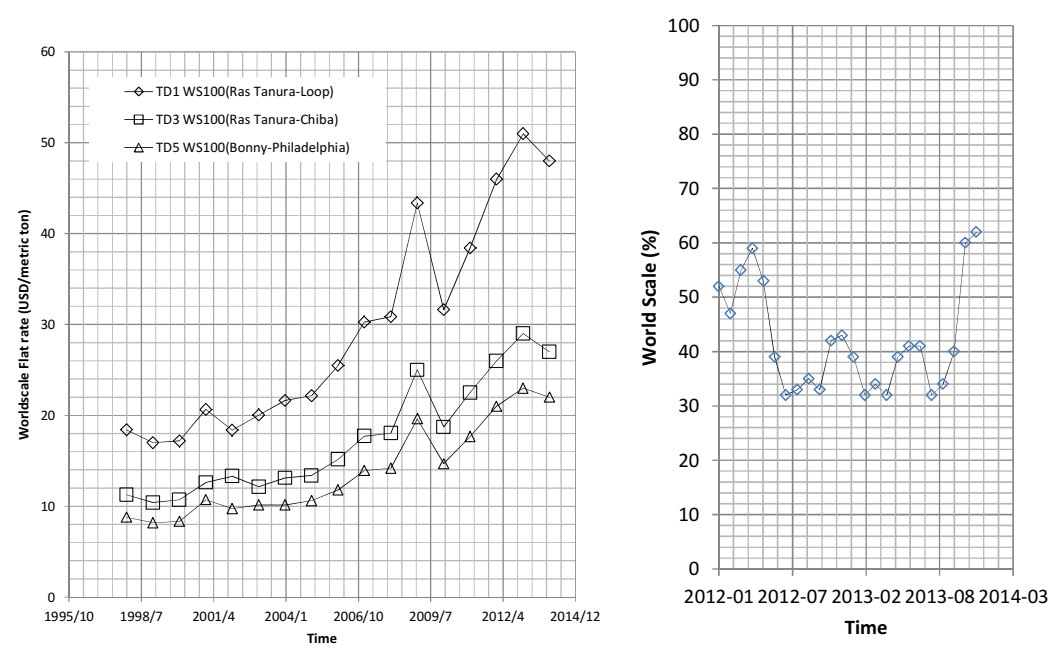

Fig. 5. Worldscale Flat Rate [5][6] and Worldscale Rate from Arabian Gulf to Japan [7] 
If a route is given, revenue the amount of and fuel consumption can be estimated by the fuel consumption model described in 2.2. The fuel cost can be calculated by the fuel consumption is if the oil price is given. The revenue can be calculated if the duration of a round trip is given by defining the service speed. Finally the cost and the revenue will tell the gross margin of a designed vessel.

\subsection{Simulation}

If a profile of a ship is given, the ship performance including the fuel oil consumption rate can be calculated. The round trip date for a voyage can be estimated by the ship speed, so the basic revenue of a ship can be calculated based on the Worldscale rate. The general flow diagram is shown in Figure 6.

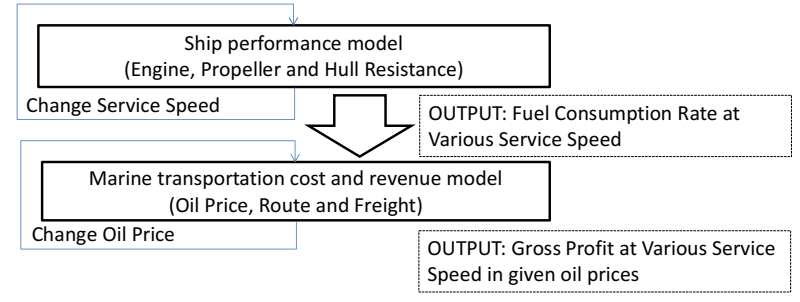

Fig. 6. General flow diagram of simulation

\section{Case Study}

This case study focuses on the requirement definition of tankers. The case illustrate potential for the improvement in terms of economic value to explore design requirement though configurations of the main engine, propellers, ship hulls and other components are optimized for a certain condition.

\subsection{Route and Ship}

In the case study, a route from Arabian Gulf to Japan is selected for detailed calculation. Basic information is shown in Table 1.

Table 1. Route overview

\begin{tabular}{|l|l|}
\hline Origin and destination & $\begin{array}{l}\text { From Ras Tanura (Arabian Gulf:AG), Saudi Arabia to } \\
\text { Chiba, Japan via Strait of Malacca }\end{array}$ \\
\hline Main Cargo & Crude oil \\
\hline Distance (one way) & 6,590 (mile) \\
\hline
\end{tabular}


Table 2. Principal particulars of the ship (based on [8])

\begin{tabular}{|c|c|}
\hline Length overall (Loa) [m] & 333 \\
\hline Length between perpendiculars (Lbp) [m] & 324 \\
\hline Breadth moulded (Bmld) [m] & 60 \\
\hline Depth moulded (Dmld) $[\mathrm{m}]$ & 29 \\
\hline Draft $[\mathrm{m}]$ & $20.529(\mathrm{~m})$ \\
\hline Deadweight [metric ton] & 300,610 \\
\hline Gross Tonnage $[\mathrm{G} / \mathrm{T}]$ & 159,960 \\
\hline Displacement [metric ton] & 341,610 \\
\hline Light weight [metric ton] & 41,000 \\
\hline Block coefficient [non dimensional] & 0.835 \\
\hline Maximum Continuous Output & $27,160 \mathrm{kw} 74.0 \mathrm{rpm}$ \\
\hline Normal Output & $23,090 \mathrm{kw} 70.1 \mathrm{rpm}$ \\
\hline Maximum velocity at sea trial & 16.74 knots \\
\hline Service Speed at Normal Output & 15.7 knots \\
\hline Diameter of propeller & $10(\mathrm{~m})$ \\
\hline Fuel consumption rate & 97.1 (t/day) \\
\hline
\end{tabular}

Table 3. Specific fuel consumption of main engine (calculated on Man Diesel \& Turbo SE web page)

\begin{tabular}{|c|c|c|c|c|c|}
\hline Load $(\%)$ & SFOC $(\mathrm{g} / \mathrm{kWh})$ & Load $(\%)$ & SFOC $(\mathrm{g} / \mathrm{kWh})$ & Load $(\%)$ & SFOC $(\mathrm{g} / \mathrm{kWh})$ \\
\hline 100 & 173.3 & 75 & 170.6 & 50 & 175.6 \\
\hline 95 & 172.2 & 70 & 171.1 & 45 & 177.2 \\
\hline 90 & 171.3 & 65 & 171.9 & 40 & 179 \\
\hline 85 & 170.6 & 60 & 172.9 & 35 & 181.1 \\
\hline 80 & 170.3 & 55 & 174.1 & & \\
\hline
\end{tabular}



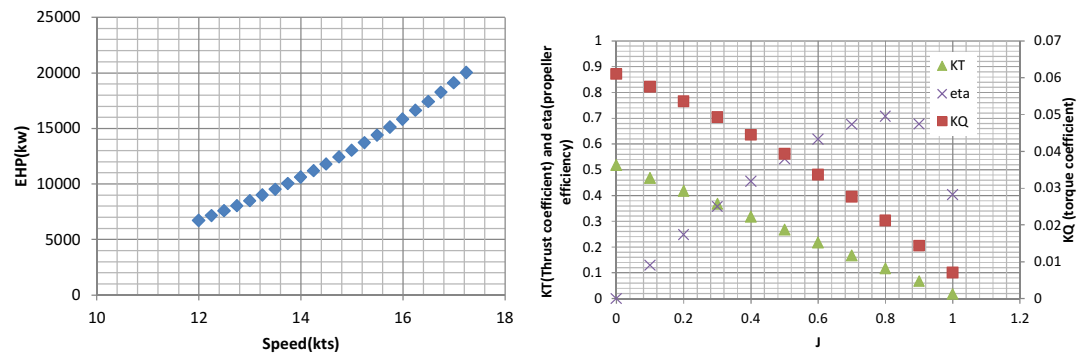

Fig. 7. Performance of Ship Hull (left) and Propeller (right)

In addition to the route, an average ship design model is also defined as shown in Table 2. This is a typical principal particulars referring to several ship design provided by shipyards and ship owners such as [8]. The specific oil consumption rate for the main engine is assumed based on the calculation provided by Man Diesel \& Turbo SE web page. The performance of the ship hull and the propeller is assumed as shown in Figure 7. Load is the ratio of actual output to maximum continuous output of the main engine. The data is not actual data though the assumption is based on several design data.

\subsection{Results}

The calculation results of the gross margin which is subtraction of revenue and cost, and the fuel oil consumption rate given by the ship performance model.

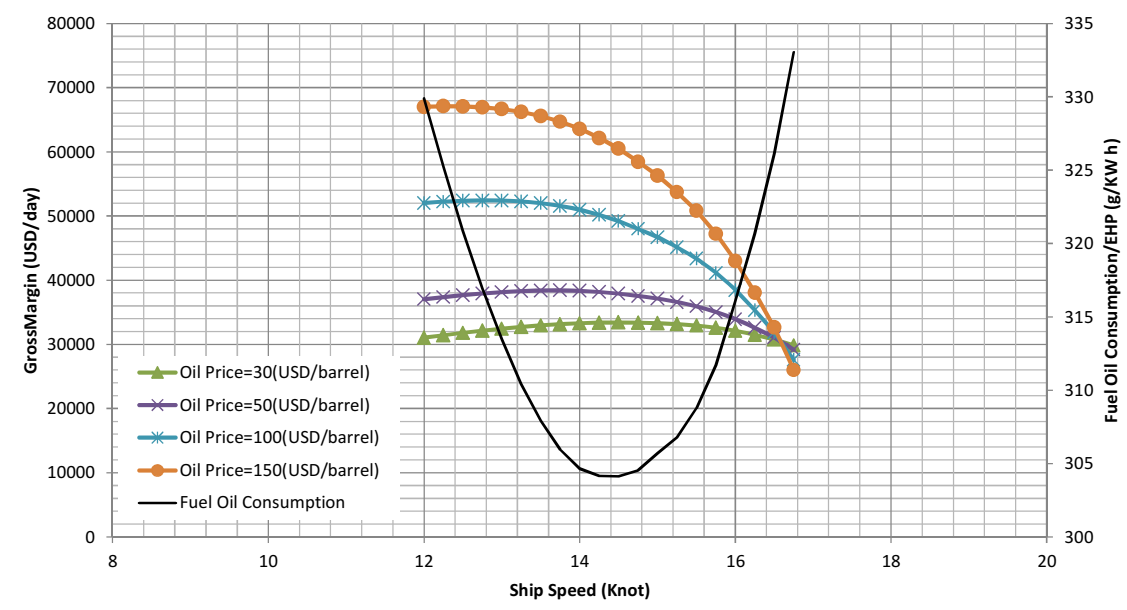

Fig. 8. Ship performance in terms of economic and technological evaluation

The revenue goes up by increasing the ship speed as the duration for a round trip of a voyage is getting smaller. The cost is getting higher because of the nonlinear increase of 
the resistance of ship hull shown as EHP. The results are shown as lines with markers in Figure 8. In this simulation, the Worldscale flat rate is projected by the oil price and the Worldscale rate for the route is fixed to WS45.

In terms of the fuel consumption rate, the main engine and the propeller have an optimal condition for operations. Ship design is usually optimized for the condition. This performance from the technological point of view is shown as a solid line in Figure 8.

The peak performance in terms of the technological perspective is found at around design service speed; however the optimal speed for the gross margin moves as the oil price changes. The oil price goes up and the optimal speed for maximizing the gross margin will getting smaller.

\section{Discussion}

The results are reasonable and explain the current marine transportation situation. The oil price is getting higher these years and slow steaming is widely deployed to decrease the cost of shipping firms. The design of ships still has to focus on the optimization in terms of the technological performance. It can be suggested that ship design should have a certain level of consideration for the economic performance to respond the uncertainties such as oil price and market in the future. This kind of idea is proposed by de Nufville [9].

Specifically, this research focuses on the evaluation of service speed and the performance with limited collected data. The detailed ship and market model and more data are required to obtain accurate simulation results.

\section{Conclusion}

A requirements evaluation method to maximize the benefit of ships in the operation is proposed and applied to a tanker traveling between Arabian Gulf and Japan. The simulation results based on the proposed method show that the optimal conditions are different in terms of economic and technological perspective. This research suggests ship design should consider the maximization against the uncertainties in economic situation because of the length of the service life.

\section{References}

1. Tsujimoto, M., Sasaki, N., Fujiwara, T., Ueno, M., Ushui, N., Kado, M., Nomura, D., Takagi, K.: A Calculation Method of 10 mode Index for Ships. Journal of the Japan Society of Naval Architects and Ocean Engineers 10, 97-104 (2009)

2. Gillmer, T.C., Johnson, B.: Introduction to Naval Architecture, U.S. Navy (1982)

3. Sanguri, M.: How to Use Main Engine Performance Curve for Economical Fuel Consumption on Ships? (August 16, 2012), http: / / www .marineinsight.com/marine/ marine-news / headline/how-to-use-main-engine-performance-curvefor-economical-fuel-consumption-on-ships / (January 20, 2014) 
4. Federal Reserve Bank of St. Louis, Crude Oil Prices: West Texas Intermediate (WTI) Cushing, Oklahoma (MCOILWTICO) (February 20, 2014), http://research. stlouisfed.org/ fred2/series / MCOILWTICO (February 28, 2014)

5. McQuilling Services, LLC, No $21-2013$ Worldscale Flat Rate Forecast, Tankers (2012)

6. McQuilling Services, LLC., No 11 - 2012 Flat Rate Forecast, Tankers (2011)

7. MOL, Market Data (February 2014), http://www.mol.co.jp/ir-e/data_e/ market_e.html (February 28, 2014)

8. IHI, Press release (July 2003), http://www.ihi.co.jp/ihi/press/2003/ 2003-7-08/index.html (February 28, 2014)

9. de Neufville, R., Scholtes, S.: Flexibility in Engineering Design. MIT Press (August 2011) 